\title{
Treatment of Poor-Grade Subarachnoid Hemorrhage Trial
}

\author{
D. Mitra, B. Gregson, V. Jayakrishnan, A. Gholkar, A. Vincent, P. White, and P. Mitchell
}

\begin{abstract}
BACKGROUND AND PURPOSE: Management of poor-grade subarachnoid hemorrhage is based on limited evidence from small singlecenter retrospective observational studies. The purpose of this study was to undertake a single-center randomized controlled feasibility trial comparing a strategy of early endovascular aneurysm treatment with treatment after neurologic recovery in this group of patients.
\end{abstract}

MATERIALS AND METHODS: Patients with poor-grade SAH were randomized within 24 hours of admission to early treatment or treatment after neurologic recovery. If a patient was randomized to early treatment, the aneurysm was treated endovascularly within 24 hours of randomization. Recruitment rate, safety profile, and functional outcome at the time of discharge and at 6 months were assessed.

RESULTS: Fourteen of 51 patients screened were eligible for the trial. Of these 14, 8 patients were randomized (57\%). All patients in the early coiling arm received treatment within 24 hours of randomization. There was no treatment-related complication. Overall, good outcome occurred in $25 \%$ of patients; the mortality rate was $75 \%$. Patients in the early treatment arm $(n=5)$ had a good outcome rate of $20 \%$, while those in treatment after neurologic recovery arm $(n=3)$ had a good outcome rate of $33.3 \%$.

CONCLUSIONS: This was a feasibility study that demonstrated that recruitment and randomization for comparing management strategies in poor-grade SAH are feasible. The recruitment rate among eligible patients was encouraging (57\%), though a number of patients had to be excluded due to ineligibility. A multicenter study is necessary to recruit the numbers required to compare the clinical outcomes of these management strategies.

ABBREVIATIONS: ISAT = International Subarachnoid Aneurysm Trial; WFNS $=$ World Federation of Neurosurgical Societies

I ntracranial aneurysms are being treated with increasing frequency by endovascular coiling. The International Subarachnoid Aneurysm Trial (ISAT), ${ }^{1}$ an international, multicenter, randomized controlled trial comparing coiling with surgical clipping, demonstrated that there was a $6.9 \%$ reduction of absolute risk and a $22.6 \%$ reduction of relative risk of death or dependency at the end of 1 year in the coiling arm. On the basis this finding, early aneurysm coiling of patients with good-grade subarachnoid hemorrhage has become an established practice. Poor-grade SAHs (World Federation of Neurosurgical Societies [WFNS] grades IV

Received February 21, 2014; accepted after revision May 19.

From the Departments of Neuroradiology (D.M., V.J., A.G.), Neurosurgery (P.M.), and Anesthesiology and Intensive Care (A.V.), Royal Victoria Infirmary, Newcastle upon Tyne, United Kingdom; and Institutes of Aging and Health (P.W.) and Neurosciences (B.G.), Newcastle University, Newcastle upon Tyne, United Kingdom.

Please address correspondence to Dipayan Mitra, MD, Department of Neuroradiology, Level Five New Victoria Wing, Royal Victoria Infirmary, Newcastle upon Tyne, NE1 4LP, United Kingdom; e-mail: Dipayan.Mitra@nuth.nhs.uk

Evidence-Based Medicine Level 2.

http://dx.doi.org/10.3174/ajnr.A4061 and V) were significantly under-represented in ISAT because these patients are usually not considered for clipping unless they have made substantial clinical improvement. ${ }^{2,3}$ Although they were not under-represented in the Barrow Ruptured Aneurysm trial, this study did not assess treatment timing. ${ }^{4}$ ISAT re-raised the question of the balance of risks of aneurysm treatment in the early days after poor-grade SAH but did not provide the data to determine the answer. ${ }^{1}$ Early coiling could potentially have the advantage of reducing the rebleeding rate without the increased treatment risks incurred by early surgical clipping in poor-grade SAH patients. ${ }^{2}$ The benefit of such a strategy, however, cannot be extrapolated from ISAT and should be supported by robust (randomized controlled trial) data before being adopted as standard practice. We have undertaken a single-center randomized parallel-group feasibility trial between patients with poor-grade SAH who were treated early with endovascular coiling and those who were managed by the traditional method (ie, treated [coiled or clipped] after clinical neurologic improvement) as a first step toward answering this important question. The outcome measures were the following: 1) recruitment rate, 2) trial safety assessed by 
mortality and adverse event rates, and 3 ) distribution of 6-month functional outcome measured by the modified Rankin Scale score. This pilot study is expected to inform the design of a future multicenter randomized controlled trial.

\section{MATERIALS AND METHODS}

Full approvals from the institutional ethics committee and hospital research and development department were obtained for the trial.

\section{Inclusion and Exclusion Criteria}

The trial included patients older than 18 years admitted to the neurosciences intensive therapy unit with WFNS grade IV or V $\mathrm{SAH}$ who were hemodynamically stable and whose next of kin provided assent for inclusion.

Exclusion criteria were the following: 1) age older than 75 years, 2) signs of brain stem death not promptly reversed by anticerebral edema treatment, 3) pure intraventricular hemorrhage, 4) large intracerebral hematoma requiring immediate clot evacuation, 5) lack of clinical equipoise (ie, the treating clinician believed that there was a much greater benefit to be gained for that patient by one or the other of the treatment arms), and 6) pregnancy.

\section{Trial Design and Randomization}

Patients admitted to the intensive therapy unit were stabilized from neurologic and cardiorespiratory points of view as per established protocol. If the patient was initially admitted to a different hospital, confirmation of the Glasgow Coma Scale score before intubation/ventilation was sought from the referral letter. Once SAH was confirmed by imaging and the patient was stable, the admitting neurosurgical/anesthetic team assessed them for eligibility for the trial.

If the patient fulfilled the eligibility criteria, an appropriate clinician (intensive therapy unit consultant/registrar or neurosurgical consultant/registrar or neuroradiology consultant/registrar) discussed the trial and provided written information to the next of kin. The clinician returned after an interval to allow adequate time for reflection and obtained informed assent for the trial from the next of kin.

If assent was not obtained from the next of kin, the reason was documented.

A screening log was completed, recording the number of patients assessed, the number meeting the inclusion criteria, and the number excluded because of the presence of $\geq 1$ exclusion criteria (and if excluded, the reason for it).

After obtaining assent, we performed randomization within 24 hours of admission to the intensive therapy unit. A randomized block design (with variable block sizes of 2, 4, and 6) was used with 2 strata (hydrocephalus and no hydrocephalus) to ensure that patients with significant hydrocephalus (as determined by a requirement for CSF drainage) were equally distributed between the 2 trial arms.

\section{The 2 Trial Arms}

Early Treatment Arm. If the patient was randomized to the early treatment arm, the result of randomization was communicated to the interventional neuroradiology team. Appropriate assent for the coiling procedure was then obtained. If amenable to endovascular treatment, the aneurysm was treated within 24 hours of randomization.

Treatment after Clinical Improvement Arm. If the patient was randomized to the treatment after clinical improvement arm, the result was communicated to the intensive therapy unit and neurosurgical team who continued managing the patient as per local established protocol. If and when the patient's neurologic status improved to WFNS grade III or better, the aneurysm was treated appropriately. There was no specific time-delay criterion for aneurysm treatment in this arm.

\section{Aneurysm Treatment}

Standard local procedures for aneurysm treatment were followed. Endovascular treatment of the aneurysms was performed with biplane angiography equipment (Integris Allura; Philips Healthcare, Best, the Netherlands). All patients were treated under general anesthetic and received a standard regimen of anticoagulation with heparin. Balloon assistance was used as required. None of the patients required a stent-assisted technique.

\section{Follow-Up}

Angiographic Follow-Up. Angiographic follow-up was performed as per the local established protocol (5 and 24 months following the coiling procedure).

Clinical Follow-Up. Patients were assessed for their functional status at the time of discharge and at 6 months following ictus. Assessment was performed by members of the endovascular team blinded to the results of randomization on the basis of the modified Rankin Scale.

\section{RESULTS}

\section{Screening and Recruitment}

The study was performed in a single United Kingdom neurosciences center. Fifty-one patients admitted to the intensive therapy unit with poor-grade SAH were screened over 29 months (August 2008 to January 2011). Fourteen patients were found to be eligible for the trial. Eight of 14 eligible were randomized for the study. The other 6 could not be included because assent from the next of kin could not be obtained. The recruitment rate among patients eligible for the trial was therefore $57 \%$, while the recruitment rate of patients screened was $16 \%$.

Thirty-seven patients were excluded from the study because they did not meet the inclusion criteria. A number of factors were responsible for patients being ineligible for the trial, which are summarized in Table 1.

Five patients were randomized to the early treatment arm, and 3 patients were randomized to the treatment after recovery arm.

\section{Demographics}

Of the 8 patients included in the study, 4 were men and 4 were women. The age of patients ranged between 26 and 64 years, with an average age of 53 years. Of the 5 patients ( 3 women and 2 men) randomized to early treatment, the age range was 26-64 years with an average age of 53 years. Of the 3 patients ( 2 men and 1 
Table 1: Reason for ineligibility for randomization among the patients screened for the trial

\begin{tabular}{lcc}
\hline \multicolumn{1}{c}{ Reason for Ineligibility } & No. & $\%$ \\
\hline $\begin{array}{l}\text { Improvement of neurologic status before } \\
\quad \text { randomization }\end{array}$ & 8 & 23 \\
Expiry of time window for randomization & 5 & 13 \\
Emergency clot evacuation & 5 & 13 \\
Hemodynamic instability & 5 & 13 \\
Beyond age range & 4 & 11 \\
Pure intraventricular hemorrhage & 3 & 8 \\
Signs of coning & 3 & 8 \\
Lack of equipoise & 3 & 8 \\
No aneurysm on CT angiogram (AVM found) & 1 & 3 \\
\hline
\end{tabular}

\section{Table 2: Treatment-unrelated adverse events}

Treatment-Unrelated Adverse Event

Aneurysm rebleeding

Hydrocephalus (early)

Hydrocephalus (late)

Intracerebral hematoma

Intraventricular hemorrhage

Diffuse cerebral edema

woman) randomized to treatment after recovery, the age range was 51-54 years, with an average age of 52 years.

\section{Prerandomization Assessment}

Five patients in the study had an admission WFNS grade of V, while 3 patients had an admission WFNS grade of IV. Of patients in the early treatment arm, 3 had a WFNS grade of $V$ and 2 had a WFNS grade of IV. Of patients in the treatment after recovery arm, 2 patients had a WFNS grade of V, while 1 had a WFNS grade of IV.

All patients included in the trial had a CT head study before randomization. The Fisher grade of the admission CT was available in 7 of the 8 patients in the trial. All these CT studies demonstrated Fisher grade $4 \mathrm{SAH}$.

\section{Randomization}

Patients were randomized within 5-24 hours of admission to the intensive therapy unit (average, 11 hours). The mean time interval between randomization and aneurysm treatment in patients randomized to the early treatment arm was 9 hours 30 minutes (range, 2 hours 15 minutes to 22 hours). Only 1 of the 3 patients randomized to the treatment after recovery arm received aneurysm treatment. The time interval between randomization and aneurysm treatment in this patient was 21 hours.

\section{Aneurysm Treatment and Adverse Events}

Six of 8 patients in the study received endovascular treatment of their aneurysms. Two patients (both in the treatment after neurologic recovery arm) died before the aneurysm was treated. None of the patients in the study received surgical clipping.

Of the patients who had endovascular aneurysm treatment, there were no treatment-related adverse events. Adverse events not related to treatment are summarized in Table 2. At the time of randomization, 5 patients ( 3 in the early treatment arm and 2 in the conventional arm) had hydrocephalus requiring surgical
Table 3: Summary of outcomes between early treatment and treatment after recovery arms

\begin{tabular}{lcc}
\hline & $\begin{array}{c}\text { Early } \\
\text { Treatment Arm }\end{array}$ & $\begin{array}{c}\text { Treatment after } \\
\text { Recovery Arm }\end{array}$ \\
\hline No. of patients & 5 & 3 \\
WFNS score on admission & WFNS IV-II & WFNS IV-I \\
& WFNS V-III & WFNS V-II \\
Good outcome & 1(MRS 1) & 1 (MRS 0) \\
Poor outcome & 4 (all MRS 6) & 2 (all MRS 6) \\
\hline
\end{tabular}

drainage. Delayed hydrocephalus occurred in 1 patient (early treatment arm).

\section{Outcomes}

Functional outcomes were assessed at the time of discharge and at 6 months following ictus with the modified Rankin Scale questionnaire. At the time of discharge, 1 patient had an mRS score of 2 and 1 had an mRS score of 3 . The other 6 patients died (mRS 6). At 6 months, 1 patient improved from mRS 2 to mRS 0 and the other patient improved from mRS 3 to mRS 1 . Therefore, the overall good outcome (mRS 0-2) rate at 6 months was $25 \%$, and the poor outcome rate (mRS 3-6) at the same time was $75 \%$. The overall mortality rate was also $75 \%$. The good outcome rate in the early treatment arm was $20 \%$, while the good outcome rate in the treatment after recovery arm was 33\% (Table 3).

\section{DISCUSSION}

Endovascular treatment is relatively less invasive than clipping and has been demonstrated to improve outcome in patients with good-grade SAH. ${ }^{1}$ As a result, a number of centers are now also treating patients with poor-grade SAH with early endovascular coiling. Several single-center, retrospective, nonrandomized studies on early endovascular treatment of patients with poorgrade SAH have been published, ${ }^{5-12}$ as summarized in Table 4. While these studies provide important data in understanding outcomes in this group of patients, there are a number of difficulties in interpreting their results. First, sizes of the studies are mostly rather modest and vary widely, from $11^{6}$ to 111 subjects. ${ }^{10}$ Second, the inclusion criteria are also extremely variable. For example, while 1 study considers WFNS grade IV and V to be poorgrade, ${ }^{12}$ another study has only included WFNS grade $\mathrm{V},{ }^{6}$ and some have included patients on the basis of Hunt and Hess grades 4 and 5..$^{5,7,8,10}$ Significant heterogeneity also exists in outcome measures and follow-up. A small majority of studies have used the Glasgow Outcome Scale over the modified Rankin Scale. The follow-up also varied between 6 months ${ }^{12}$ to up to 2 years. ${ }^{10}$ There are also differences in the definition of good and poor outcomes. $^{5-12}$

With regard to neurosurgical studies, the International Cooperative Study ${ }^{2}$ reported a good recovery rate in patients with poorgrade $\mathrm{SAH}$ ranging from $10 \%$ to $33 \%$ in the various subgroups based on the timing of surgery. Some smaller scale single-center studies suggested that early surgical clipping may lead to better results, but mostly in selected groups. ${ }^{3,13,14}$ A study that included patients with poor-grade SAH who did not receive aneurysm treatment (ie, neither clipping nor coiling) reported a mortality rate of $71 \% .^{15}$

Specifically lacking from the endovascular studies are data 
Table 4: Summary of studies of patients with poor-grade subarachnoid hemorrhage treated with early endovascular coiling

\begin{tabular}{|c|c|c|c|c|}
\hline Study (yr) & $\begin{array}{l}\text { No. of } \\
\text { Patients }\end{array}$ & Methodology & $\begin{array}{c}\text { Good } \\
\text { Outcome (\%) }\end{array}$ & $\begin{array}{l}\text { Mortality } \\
\text { Rate (\%) }\end{array}$ \\
\hline Bracard et $\mathrm{al}^{5}(2002)$ & 80 & $\begin{array}{l}\text { Included HH grades } 4 \text { and } 5 \text {, good outcome GOS } 1 \text { or } 2 \text {, } \\
\text { up to } 1 \text { year follow-up }\end{array}$ & 52.5 (GOS 1-2) 8.75 GOS 3 & 37.5 \\
\hline van Loon et $\mathrm{al}^{6}(2002)$ & 11 & $\begin{array}{l}\text { WFNS grade V, outcome measure GOS, mean follow-up } \\
12 \text { months }\end{array}$ & 36 & 18 \\
\hline Weir et $\mathrm{al}^{7}(2003)$ & 27 & $\begin{array}{l}\text { HH grades } 4 \text { and } 5 \text {, outcome assessed by mRS, mean follow-up } \\
23 \text { months }\end{array}$ & 30 & 59 \\
\hline Jain et $\mathrm{al}^{8}(2004)$ & 13 & $\begin{array}{l}\text { Older than } 70 \mathrm{yr} \text {, poor } \mathrm{HH} \text { grades } 4 \text { and } 5 \text {, outcome } \\
\text { measure GOS }\end{array}$ & 15 & 47 \\
\hline Bergui and Bradac $^{9}(2004)$ & 45 & & 48 & 44 \\
\hline Suzuki et $\mathrm{al}^{10}(2006)$ & 111 & $\mathrm{HH}$ grades $4 / 5$, outcome by mRS, follow-up $\leq 24$ mo & 35.1 & 32.4 \\
\hline Pereiera et al ${ }^{11}$ (2007) & 51 & $\begin{array}{l}\text { Outcome by GOS at } 12 \text { mo, GOS } 1-3 \text { considered good; } \\
4-5 \text {, poor }\end{array}$ & 57 & 35 \\
\hline Taylor et $\mathrm{al}^{12}(2011)$ & 47 & WFNS grades IV/V, 6-month GOS for outcome & 53 & 27 \\
\hline
\end{tabular}

Note:-GOS indicates Glasgow Outcome Scale; HH, Hunt and Hess.

analogous to the evidence base for early clipping, which compares coiling with conservative treatment; and the argument largely depends on the extrapolated assumption that coiling is a benign intervention. While the studies mentioned inform clinical practice in poor-grade SAH to a limited extent, significant uncertainties still exist, and robust evidence in the form of a randomized controlled trial is lacking.

The present study demonstrates that recruitment to such a randomized controlled trial is feasible. The recruitment rate among patients eligible for the study was encouraging (57\%). The recruitment rate among the total number of patients screened for the study was much more modest at $16 \%$. Screening and recruitment in the current study were largely performed during normal working hours, thus excluding a number of patients outside these hours. As shown in Table 1, the most frequent reasons for ineligibility (36\%) were the following: 1) expiry of the time window for randomization, and 2) improvement of neurologic status before the patient could be randomized. If patient screening and recruitment are supported by the research staff 7 days a week, a higher recruitment rate would be realistic.

The study set out to assess the safety of conducting a randomized controlled trial in this group of patients. Six of 8 patients in the study underwent endovascular aneurysm treatment, while the other 2 died before aneurysm treatment. None of the patients undergoing endovascular treatment had any treatment-related complication; this finding suggests that a trial such as the present one is safe to conduct. As expected, a proportionately high number of disease-related adverse events did occur (Table 2). With regard to the feasibility of performing early coiling within the stipulated timeframe, the average time from randomization to treatment in patients in the early treatment arm was 9 hours 30 minutes, which suggests that the trial requirement of a maximum of 24 hours between randomization and treatment is achievable. No patient in this study had to be excluded because of noncompliance to this requirement.

Hydrocephalus is often thought to be the cause of incorrect WFNS grading and, therefore, incorrect classification as poorgrade SAH. To identify "true" poor-grade SAH, some centers advocate sedation-reversal followed by formal neurologic assessment. ${ }^{3}$ However sedation-reversal increases the risk of aneurysm rebleed, and postreversal neurologic assessment introduces delay into the process of randomization and treatment, making it diffi- cult to study the benefits of early treatment. In this study, the decision regarding sedation-reversal has been left to the intensive therapy unit team. If a patient's postreversal neurologic status improved rapidly to the extent that he or she no longer had a poor-grade $\mathrm{SAH}$, then the patient was not randomized. If a patient was randomized, then he or she remained in the allocated trial arm regardless of the speed of recovery. Hydrocephalus was used as a stratification criterion to ensure that an equal proportion of patients with hydrocephalus were included in the $2 \mathrm{arms}$ and that the outcomes were not skewed by this factor.

The overall mortality rate in this study was $75 \%$, which is higher than that in many reported series. This is most probably due to the small size of this study and possible bias toward randomizing more patients with grade $\mathrm{V}$ than with grade IV. Most patients in this study had admission WFNS grades V; and 5 of 6 patients who died had an admission WFNS grade of V.

Although the numbers are small, our experience suggests that the benefit of early endovascular treatment in a poor-grade SAH population should not be assumed unless there are robust multicenter randomized controlled trial data to support it. In this study, the good outcome rate in the treatment after recovery arm $(33 \%)$ was better than that in the early coiling arm $(20 \%)$.

\section{CONCLUSIONS}

This study suggests that a randomized controlled trial to evaluate the best management strategy in patients with poor-grade SAH is safe and feasible. While the recruitment rate among eligible patients was encouraging, a significant number of patients could not be randomized due to expiry of the time window for randomization and lack of assent. A fully resourced multicenter study should be able to address these issues and recruit sufficient numbers to achieve robust outcome data.

\section{ACKNOWLEDGMENTS}

We acknowledge the contribution of Elaine McColl, Director of Newcastle Clinical Trials Unit, and Nicola Hind, Consultant Neuroradiographer, Royal Victoria Infirmary, Newcastle upon Tyne, for their exceptional help in design and recruitment of this trial.

Disclosures: Anil Gholkar-UNRELATED: Consultancy: Stryker Neurovascular, Codman Neurovascular, MicroVention, Comments: I organize and participate in training 
courses organized by these entities; Travel/Accommodations/Meeting Expenses Unrelated to Activities Listed: Stryker Neurovascular, MicroVention, Comments: These entities have contributed to my expenses towards travel and accommodation to participate in Society of Neuro-Interventional Surgery meeting in 2013 and World Federation of Interventional and Therapeutic Neuroradiology meeting in 2011. Vijay Jayakrishnan-UNRELATED: Payment for Lectures (including service on Speakers Bureaus): Codman Depuy, Comments: for providing teaching to health care professionals. Philip White-UNRELATED: Consultancy: Codman, MicroVention, Comments: organizing educational meetings; Grants/Grants Pending: Codman, ${ }^{*}$ Covidien, ${ }^{*}$ Comments: paid to the University of Glasgow but Co-Chief Investigator on grant part industry-funded; Payment for Lectures (including service on Speakers Bureaus): Covidien; Travel/Accommodations/Meeting Expenses Unrelated to Activities Listed: MicroVention, Comments: support to attend Society of Neuro-Interventional Surgery 2013 meeting in Miami; OTHER RELATIONSHIPS: A grant for a multicenter trial has been developed and submitted to UK National Institutes of Health Research based on a feasibility pilot described in this work. Patrick MitchellUNRELATED: Grants/Grants Pending: UK National Institute for Health Research,* Comments: We are applying for a grant from National Institute for Health Research UK to run a larger version of the trial (Treatment of Poor-Grade Subarachnoid Hemorrhage Trial 2). *Money paid to the institution.

\section{REFERENCES}

1. Molyneux AJ, Kerr RS, Yu LM, et al, for the International Subarachnoid Aneurysm Trial (ISAT) Collaborative Group. International subarachnoid aneurysm trial (ISAT) of neurosurgical clipping versus endovascular coiling in 2143 patients with ruptured intracranial aneurysms: a randomised comparison of effects on survival, dependency, seizures, rebleeding, subgroups, and aneurysm occlusion. Lancet 2005;366:809-17

2. Kassell NF, Torner JC, Haley EC, et al. The international cooperative study on the timing of aneurysm surgery. Part 2 . Surgical results. J Neurosurg 1990;73:37-47

3. Hutchinson PJ, Power DM, Tripathi P, et al. Outcome from poor grade aneurysmal subarachnoid haemorrhage: which poor grade subarachnoid haemorrhage patients benefit from aneurysm clipping? Br J Neurosurg 2000;14:105-09

4. McDougall CG, Spetzler RF, Zabramski JM, et al. The Barrow ruptured aneurysm trial. J Neurosurg 2012;116:135-44
5. Bracard S, Lebedinsky A, Anxionnat R, et al. Endovascular treatment of Hunt and Hess grade IV and V aneuryms. AJNR Am J Neuroradiol 2002;23:953-57

6. van Loon J, Waerzeggers Y, Wilms G, et al. Early endovascular treatment of ruptured cerebral aneurysms in patients in very poor neurological condition. Neurosurgery 2002;50:457-64

7. Weir RU, Marcellus ML, Do HM, et al. Aneurysmal subarachnoid hemorrhage in patients with Hunt and Hess grade 4 or 5 : treatment using the Guglielmi detachable coil system. AJNR Am J Neuroradiol 2003;24:585-90

8. Jain R, Deveikis J, Thompson B. Endovascular management of poorgrade aneurysmal subarachnoid hemorrhage in geriatric population. AJNR Am J Neuroradiol 2004;25:596-600

9. Bergui M, Bradac G. Acute endovascular treatment of ruptured aneurysms in poor-grade patients. Neuroradiology 2004;46:161-164

10. Suzuki S, Jahan R, Duckwiler GR, et al. Contribution of endovascular therapy to the management of poor-grade aneurysmal subarachnoid hemorrhage: clinical and angiographic outcomes. $\mathrm{JNeu}$ rosurg 2006;105:664-70

11. Pereiera A, Sanchez-Peña P, Biondi A, et al. Predictors of 1 year outcome after coiling for poor-grade subarachnoid hemorrhage. Neurocrit Care 2007;7:18-26

12. Taylor C, Robertson F, Brealey D, et al. Outcome in poor grade subarachnoid hemorrhage patients treated with acute endovascular coiling of aneurysms and aggressive intensive care. Neurocrit Care 2011;14:341-47

13. Laidlaw JD, Siu KH. Poor-grade aneurysmal subarachnoid hemorrhage: outcome after treatment with urgent surgery. $\mathrm{Neu}$ rosurgery 2003;53:1275-80

14. Bailes JE, Spetzler RF, Hadley MN, et al. Management morbidity and mortality of poor-grade aneurysm patients. J Neurosurg 1990;72: $559-66$

15. Hijdra A, van Gijn J, Nagelkerke NJ, et al. Prediction of delayed cerebral ischemia, rebleeding, and outcome after aneurysmal subarachnoid hemorrhage. Stroke 1988;19:1250-56 